\title{
Interferon-gamma release assays for tuberculosis screening of healthcare workers: a systematic review
}

\author{
Alice Zwerling, ${ }_{1}^{1}$ Susan van den Hof, ${ }^{2,3}$ Jerod Scholten, ${ }^{2}$ Frank Cobelens, ${ }^{2,3}$ \\ Dick Menzies, ${ }^{1}$ Madhukar Pai ${ }^{1}$
}

- Additional material and appendix tables $\mathrm{A} 1$ and $\mathrm{A} 2$ are published online only. To view these files please visit the journal online (http://thorax.bmi.com/ content/67/1.toc)

${ }^{1}$ McGill University and Montreal Chest Institute, Montreal,

Canada

${ }^{2}$ KNCV Tuberculosis Foundation,

The Hague, The Netherlands

${ }^{3}$ Center for Poverty-related

Communicable Diseases,

Amsterdam Institute for Global Health and Development and

Center for Infection and Immunity Amsterdam,

Academic Medical Center,

Amsterdam, The Netherlands

\section{Correspondence to}

Madhukar Pai, Department of Epidemiology, Biostatistics and Occupational Health, McGill University, 1020 Pine Avenue West, Montreal H3A 1A2,

Canada;

madhukar.pai@mcgill.ca

The findings of this review were presented at a WHO Expert Group Meeting on interferon-gamma release assays in July 2010 organised by the Stop TB Department of the WHO

Received 25 May 2010 Accepted 2 December 2010 Published Online First 12 January 2011

\section{ABSTRACT}

Healthcare workers (HCWs) are at increased risk of exposure to tuberculosis (TB). Traditionally, screening for latent TB infection (LTBI) is done using the tuberculin skin test (TST). Interferon-gamma release assays (IGRAs) are now increasingly being used for diagnosis of LTBI, but their role in HCW screening is unclear. A systematic review was conducted of all IGRA studies in HCWs to summarise their performance in cross-sectional and serial testing settings. By searching four electronic databases and other sources, all available studies using any one of the commercial IGRA assays in HCWs were retrieved and screened. 50 unique studies were identified which met the inclusion criteria including five from high TB incidence settings. Among 24 cross-sectional studies in low TB incidence settings, the pooled prevalence of positive IGRA using either test was significantly lower than for a positive TST. However, in high-incidence settings $(n=2)$ there were no consistent differences in the prevalence of positive tests. IGRAs showed good correlation with occupational risk factors for TB exposure in low-incidence settings. Only 10 studies assessed use of IGRA for serial testing and all showed large variation in the rates of conversions and reversions, with no data suggesting that IGRAs are better at identifying the incidence of new TB infection than the TST. The use of IGRAs instead of TST for one-time screening may result in a lower prevalence of positive tests and fewer HCWs who require LTBI treatment, particularly in low TB incidence settings. However, the use of IGRAs for serial testing is complicated by lack of data on optimum cut-offs for serial testing and unclear interpretation and prognosis of conversions and reversions. Further longitudinal research will be required to inform guidelines on serial testing using IGRAs.

\section{INTRODUCTION}

Tuberculosis (TB) continues to have a significant health impact worldwide with an estimated onethird of the world's population infected with latent TB (LTBI). TB poses a significant occupational health problem and healthcare workers (HCWs) are at increased risk of exposure to TB. ${ }^{12}$ A systematic review of 51 studies showed that the prevalence and incidence of TB infection and disease were high among HCWs in low and middle income countries. ${ }^{1}$ The emergence of extensively drug-resistant TB has resulted in a renewed interest and emphasis on the protection of the healthcare workforce. The World Health Organization (WHO) recently issued a policy on TB infection control (TBIC) in resourcelimited settings and is now actively promoting TBIC programmes. ${ }^{3}$
In many high-income countries, periodic screening of HCWs for LTBI is an important component of TBIC programmes. ${ }^{4}$ Traditionally, the prevalence of LTBI and incidence of new TB infection (ie, conversion) among HCWs has been estimated using the tuberculin skin test (TST), a test with known limitations. ${ }^{5-7}$ Recently, interferon-gamma release assays (IGRAs) have emerged as alternatives for the diagnosis of LTBI. ${ }^{8-10}$ Two IGRAs are commercially available-the QuantiFERON-TB Gold In-Tube (OFT) assay (Cellestis Ltd, Carnegie, Australia) and the T-SPOT.TB assay (Oxford Immunotec, Abingdon, UK). With the development of new national guidelines incorporating IGRAs, their use is steadily increasing. ${ }^{11}$

IGRAs have features that make them attractive for repeated screening: they are ex vivo blood-based tests that, in contrast to the TST, can be repeated any number of times without sensitisation or boosting, they require only one visit and do not need a baseline two-step protocol.

There is strong evidence from systematic reviews that IGRAs, especially OFT, have excellent specificity that is unaffected by BCG vaccination, while the T-SPOT.TB shows improved sensitivity for active TB over both the TSTand QFT. ${ }^{712}{ }^{13}$ However, reviews have suggested that IGRA performance differs in high versus low TB incidence settings, with relatively lower sensitivity in high-incidence countries. $^{8} 14$

Despite the substantial body of literature on IGRAs, almost all the available studies have limitations-namely, lack of a gold standard for LTBI, cross-sectional design, use of sensitivity and specificity as surrogates for patient-important outcomes, and lack of adequate data on predictive/prognostic value of IGRAs. In particular, data are lacking on how to interpret repeated (serial) IGRA testing results. ${ }^{15}{ }^{16}$ Currently, no guidelines exist on the use of IGRAs in countries with a high incidence of TB. Some guidelines from high-income low-incidence countries have not recommended IGRAs for serial testing of $\mathrm{HCWs}^{17}$ while others state that IGRAs may be used for serial testing of HCWs in place of the TST. ${ }^{4} 9$

\section{METHODS}

Our objective was systematically to review all studies using IGRA to test HCWs, including crosssectional, longitudinal and serial testing studies, to summarise their performance characteristics. Secondary objectives included (1) to compare IGRA performance in HCWs in high or low TB incidence settings; (2) to determine if IGRAs are better 
correlated with occupational exposure to TB than the TST in cross-sectional studies; (3) to estimate the rate of IGRA conversions and reversions and assess whether IGRA conversions are more closely associated with recent occupational exposure than TST conversions; and (4) to summarise the evidence produced by cost-effectiveness analyses and programmatic studies.

\section{Data sources and searches}

We have previously published systematic and narrative reviews on IGRA accuracy and performance in various subgroups. ${ }^{10} 121318$ We updated the database searches used in previous systematic reviews and searched the literature for relevant IGRA studies. PubMed, Embase and Biosis and Web of Science were searched and citations of all original articles published in all languages up to 30 April 2010 that reported data on IGRA performance in HCWs (updated again on 1 October 2010 to include the most recent serial testing studies) were reviewed.

In addition to database searches we reviewed bibliographies of previous reviews and guidelines on IGRAs, conference proceedings, abstracts and also screened the citations of relevant original articles. Experts in the field and commercial test manufacturers were contacted to obtain relevant citations. Authors of primary studies were contacted to obtain additional information where necessary. The detailed search string and a list of conferences reviewed for relevant citations are available in the online supplement.

\section{Outcomes evaluated}

A major challenge for studies evaluating the performance of IGRAs is the lack of a gold standard for LTBI. We therefore developed a priori a hierarchy of reference standards for the performance of IGRAs in diagnosing LTBI in HCWs (figure 1). At the time of this review, there was no evidence at the highest two levels of the hierarchy nor were there studies looking at sensitivity and specificity for active TB in HCWs. We therefore evaluated studies that reported one or more of the following outcomes: prevalence and incidence of LTBI; correlation between IGRA results and an exposure gradient; and/or agreement between IGRA and TST results.

Details of study selection, inclusion and exclusion criteria, data extraction procedure and quality assessment are given in the online supplement.

\section{Data synthesis and analysis}

Study characteristics and results are presented as tables and plots. In order to compare prevalence estimates for the tests (IGRAs

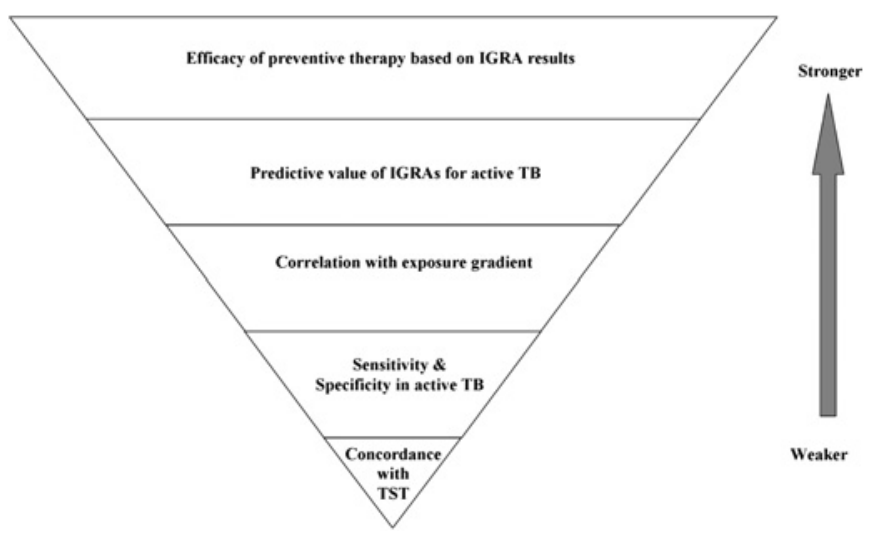

Figure 1 Hierarchy of reference standards for performance of interferon-gamma release assays (IGRAs) for diagnosing latent tuberculosis infection (LTBI) in healthcare workers. TST, tuberculin skin test. vs TST) we calculated Fisher exact 95\% CIs for the prevalence estimates extracted from the original reports. If the $95 \%$ CIs did not overlap, differences between proportions were considered as statistically significant, a conservative approach. For the association between occupational risk factors and IGRA we extracted ORs reported by the original authors. When available we extracted both crude and adjusted ORs. Because the included studies varied greatly in their design, execution and outcomes, and because meta-analysis methods are not well defined for such heterogeneous diagnostic studies with no gold standard, we did not perform a meta-analysis. IGRA performance varies across populations ${ }^{8}$ so all the results were stratified by TB incidence in the country where the study was done (high vs intermediate and low incidence). High-incidence countries were defined as countries with more than 100 estimated incident TB cases per year per 100000 population as determined by the WHO. ${ }^{19}$

\section{RESULTS}

\section{Description of included studies}

Figure 2 shows a flow chart on study selection. The final tables included 42 IGRA studies in HCWs that reported one of our main outcomes of interest. In addition we identified three studies on cost-effectiveness of IGRAs in HCWs and three studies on feasibility and test implementation. Finally, two new serial testing studies were identified by 1 October 2010, giving a total of 50 studies of IGRAs in HCWs. ${ }^{20}{ }^{21}$ The characteristics of the cross-sectional and serial testing studies identified in this review are shown in tables 1 and 2, respectively. Complete details on study methodology and test performance are given in appendix tables A1 and A2, respectively, in the online supplement. Data are presented stratified by high versus low and moderate TB incidence settings but, even within these strata, the study populations included HCWs with varying risk of $\mathrm{TB}$ exposure.

Of the 44 included studies reporting a main outcome, 35 (79\%) evaluated OFT only and 3 (7\%) used T-SPOT.TB only, while the remaining $6(14 \%)$ evaluated both IGRAs. While most studies performed both an IGRA and the TST, 14\% of studies only performed IGRA testing and therefore could not compare IGRA results with TST results. Overall, only 5 (11\%) were done in high-incidence settings. Only 10 (23\%) used a longitudinal or serial testing design. Study sizes ranged from 12 to 1313 HCWs, for a total of 11963 HCWs across the 44 studies. Most included BCG-vaccinated HCWs; however, the proportion of BCG-vaccinated HCWs varied considerably (7-100\%).

\section{Results of cross-sectional IGRA studies}

IGRA versus TST positivity rates in HCW populations

in high-incidence countries

Three cross-sectional studies evaluated IGRA performance in HCWs in India, Russia and Vietnam, although TST was not performed in the Russian study. ${ }^{22-24}$ As shown in figure 3, TST and IGRA positivity rates were high in HCWs (40-66\%); IGRA positivity was slightly lower than TST positivity in the studies in India and Vietnam comparing TST and IGRAs, but the difference in estimated prevalence between the two tests was significant only in Vietnam. ${ }^{24}$ The Vietnamese study also reported the lowest rate of BCG vaccination among its participants at $37.3 \%$ compared with $71 \%$ in the Indian study.

IGRA versus TST positivity rates in HCW populations in low- and moderate-incidence countries

We identified 31 cross-sectional studies from low or intermediate TB incidence countries. ${ }^{25-52}{ }^{54-56}$ Twenty-five studies used only 
Figure 2 Flow chart of studies included and excluded at each stage of the review. IGRA, interferon-gamma release assay.

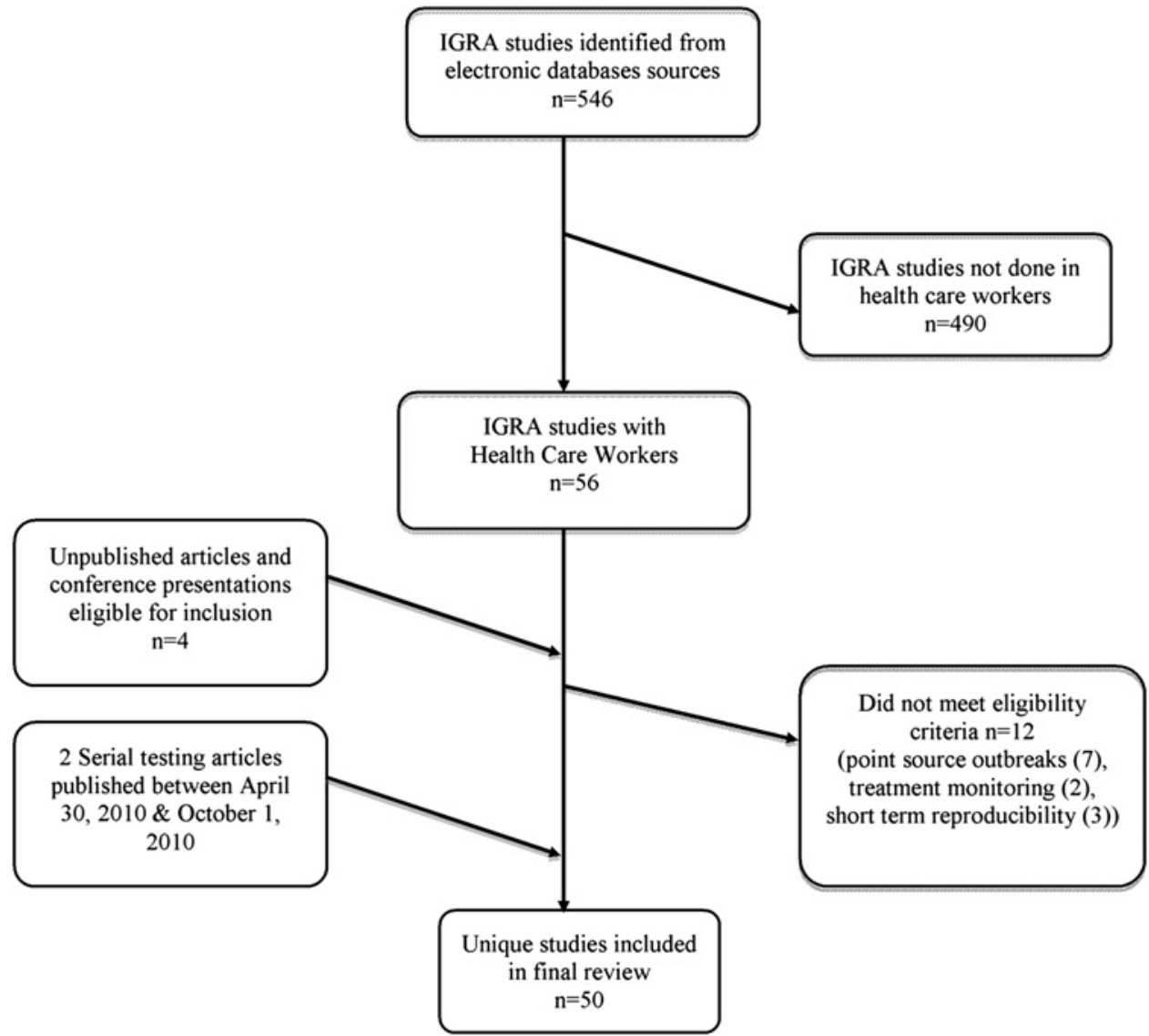

the OFT-Gold test, 2 studies only the T-SPOT.TB and 4 performed head-to-head comparisons of the OFT and T-SPOT. TB tests. ${ }^{41} 445055$ The prevalence of positive OFT ranged from $1 \%$ to $66.8 \%$ and of positive T-SPOT.TB tests from $1 \%$ to $60 \%$. Among 25 studies that compared IGRA with TST, all but one ${ }^{44}$ reported a lower prevalence of positive OFT or T-SPOT.TB than positive TST (figures 3 and 4), with statistically significant differences found in 17 of these 24 studies. The studies in figure 4 are shown in order of increasing proportion of participants BCG vaccinated; studies in which a higher proportion of study participants were BCG vaccinated did not necessarily have a higher prevalence of positive TST or a larger difference between the prevalence of positive TST and positive IGRA.

Concordance was weak between TST and IGRAs in these studies with $\kappa$ values ranging widely from 0.05 in Denmark ${ }^{29}$ (using a $12 \mathrm{~mm}$ TSTcut-off) to 0.56 in a study from Spain $^{49}$ (using a $15 \mathrm{~mm}$ TST cut-off for BCG-vaccinated individuals). Three studies evaluated a range of TST cut-offs in the analysis and all showed that agreement could be improved by employing a more stringent TST cut-off (ie, $15 \mathrm{~mm}$ vs $10 \mathrm{~mm}$ ). ${ }^{33} 4647$ In all 19 studies that reported on discordance, TST +/IGRA - subjects was the predominant type of discordance. ${ }^{25} 272932-35373946-5254-56$

In summary, among these 34 cross-sectional studies the prevalence of positive IGRA was lower than positive TST. The difference in prevalence was significant in low and moderate TB incidence settings (figure 4) but not in high-incidence settings.

\section{Association between occupational risk factors and test results in HCW populations}

The majority of studies reporting such analyses were done in low-incidence settings. Of three cross-sectional studies conducted in high-incidence settings, only Pai et al evaluated associations between occupational risk factors and both TST and IGRA. They found a stronger but non-significant association between occupational risk factors and IGRA positivity than the TST. $^{22}$

Of the 31 cross-sectional studies conducted in low-incidence countries, 22 evaluated potential risk factors for IGRA and/or TST reported a positive association between IGRA positivity and occupational risk factors, including higher risk for clinical staff working in a high-risk ward, TB clinic or geriatric care and increased duration of healthcare employment. Two studies ${ }^{38} 39$ reported no association between test positivity and risk factors; the remaining studies either did not perform the TST or did not calculate ORs for risk factors associated with test positivity. The age-adjusted ORs for TST and IGRAs and occupational risk factors for relevant studies are compared in figure 5; all studies are from low- and moderate-incidence countries with the exception of the India study. All three tests correlated well with established indicators of occupational risk of $\mathrm{TB}$ exposure, although no test was consistently more often associated with these indicators of exposure. Being of foreign birth or having lived in a high TB incidence country was correlated with OFT positivity in four studies, ${ }^{35} 404648$ and three of the four studies also showed correlation with TST positivity while the fifth study to investigate foreign birth as a risk factor-and the only study to do this using the TSPOT.TB test-found no association with place of birth although there was an association for TST. ${ }^{39}$ From these cross-sectional studies, IGRAs appear to be well correlated with TB infection risk factors (including occupational risk factors) in HCWs in low- and intermediate-incidence countries. positivity rates. Fourteen studies 2630323536404243464950525557 
Table 1 Characteristics of 34 cross-sectional IGRA studies in HCWs

\begin{tabular}{|c|c|c|c|c|c|c|}
\hline Study & Year & Country & $\mathbf{N}$ & TST (PPD dose) & IGRA (QFT, TSPOT.TB or both) & BCG vaccinated (\%) \\
\hline \multicolumn{7}{|c|}{ High TB incidence countries } \\
\hline Pai et $a l^{22}$ & 2005 & India & 726 & $1 \mathrm{TU}$ & OFT-G-IT & 71 \\
\hline Drobniewski et $a l^{23}$ & 2007 & Russia & 500 & - & QFT-G-IT & 84 \\
\hline Lien et $a l^{24}$ & 2009 & Vietnam & 300 & $5 \mathrm{TU}$ & QFT-G-IT & 37.3 \\
\hline \multicolumn{7}{|c|}{ Intermediate and low TB incidence countries } \\
\hline Kang et $a l^{25}$ & 2005 & Republic of Korea & 171 & $2 \mathrm{TU}$ & QFT & 92.3 \\
\hline Harada et $a l^{26}$ & 2006 & Japan & 332 & $2.5 \mathrm{TU}$ & QFT-G & 91.3 \\
\hline Ozekinci et $a l^{27}$ & 2007 & Turkey & 66 & $5 \mathrm{IU}$ & T-SPOT.TB & 67 \\
\hline Veeser et $a l^{28}$ & 2007 & USA & 55 & - & QFT-G & 45 \\
\hline Soborg et $a l^{29}$ & 2007 & Denmark & 139 & $2 \mathrm{TU}$ & QFT-G & 76 \\
\hline Nienhaus et $a l^{30}$ & 2007 & Germany & 454 & - & QFT-G-IT & 42 \\
\hline Nienhaus et $a l^{31}$ & 2007 & Germany & 161 & $2 \mathrm{TU}$ & QFT-G-IT & 36 \\
\hline Mirtskhulava et $a l^{32}$ & 2008 & Georgia & 265 & $5 \mathrm{TU}$ & QFT-G-IT & 77.7 \\
\hline Hotta et $a l^{33}$ & 2007 & Japan & 207 & $3 \mathrm{TU}$ & QFT-TB-2G & $92 \%$ (48\% with $>1$ BCG) \\
\hline Nienhaus et $a l^{34}$ & 2008 & Germany & 261 & $2 \mathrm{TU}$ & QFT-G-IT & 37.5 \\
\hline Ciaschetti et $a l^{35}$ & 2007 & Italy & 590 & - & QFT-G & 56 \\
\hline Eum et $a l^{36}$ & 2008 & Republic of Korea & 73 & - & QFT-GIT & 100 \\
\hline Choi et $a l^{37}$ & 2008 & Republic of Korea & 84 & $2 \mathrm{TU}$ & OFT-G & 100 \\
\hline Carvalho et $a l^{38}$ & 2008 & Italy & 65 & $5 \mathrm{IU}$ & QFT-G & 85 \\
\hline Barsegian et $a /^{39}$ & 2008 & Germany & 95 & - & T-SPOT.TB & 36 \\
\hline Stebler et $a l^{40}$ & 2008 & Switzerland & 777 & - & QFT-G-IT & 87.4 \\
\hline Thijsen et $a l^{41}$ & 2008 & The Netherlands & $19 *$ & - & QFT-G-IT and T-SPOT.TB & 16 \\
\hline Demkow et al ${ }^{42}$ & 2008 & Poland & 155 & - & QFT-G & 100 \\
\hline Schablon et al ${ }^{43}$ & 2009 & Germany & 270 & - & QFT-G-IT & 52.8 \\
\hline Dorman et $a l^{44}$ & 2009 & USA & 1313 & - & QFT-G-IT and T-SPOT.TB & - \\
\hline Mehta et $a l^{45}$ & 2009 & USA & $12 \dagger$ & & QFT-G & - \\
\hline Vinton et $a l^{46}$ & 2009 & Australia & 481 & $10 \mathrm{IU}$ & OFT-G-IT & 78 \\
\hline Zrinski Topić et al ${ }^{47}$ & 2009 & Croatia & 54 & $2 \mathrm{TU}$ & OFT-G-IT & 100 \\
\hline Khanna et $a l^{48}$ & 2009 & UK & 171 & $2 \mathrm{TU}$ & OFT-G-IT & 82.5 \\
\hline Álvarez-León et $a{ }^{49}$ & 2009 & Spain & 134 & $2 \mathrm{TU}$ & QFT-G-IT & 35 \\
\hline Casas et $a l^{50}$ & 2009 & Spain & 147 & $2 \mathrm{TU}$ & T-SPOT.TB \& OFT-G-IT & 16 \\
\hline Fox et $a l^{51}$ & 2009 & Israel & 100 & 5 PPD & QFT-G-IT & 37 \\
\hline Costa et $a l^{5253 *}$ & 2009 & Portugal & 1218 & $2 \mathrm{TU}$ & QFT-G-IT & 100 \\
\hline Zhao et $a l^{54}$ & 2009 & USA & 40 & - & QFT-G-IT & - \\
\hline Girardi et $a l^{55}$ & 2009 & Italy & 115 & $5 \mathrm{IU}$ & T-SPOT.TB and QFT-G-IT & 37.4 \\
\hline Cummings et $a l^{56}$ & 2009 & USA & 182 & - & QFT-G-IT & 7 \\
\hline
\end{tabular}

*Two studies based on same cohort, ERJ data displayed in table.

†All subjects were TST converters at recruitment.

BCG, Bacille Calmette-Guerin vaccine; HCW, healthcare worker; IGRA, interferon-gamma release assay; IU, international unit; PPD, purified protein derivative; OFT, OuantiFERON test; OFT-G, OFT Gold test; QFT-G-IT; QFT Gold In-Tube test; TB, tuberculosis; TST, tuberculin skin test; TU, tuberculin unit.

\section{Longitudinal serial testing IGRA studies and their results}

IGRA conversion and reversion rates in HCW populations

in high-incidence countries

We identified only two serial testing studies from high-incidence settings. Studies conducted repeat testing at 0,6 and
12 months ${ }^{58}$ and 0 and 18 months (table 2). ${ }^{15}$ The rates of IGRA conversions from these two studies ranged from $11.6 \%$ to $21 \%$. The study by Pai et al was the only study to calculate the TST conversion rate and found a $4 \%$ rate after 18 months. ${ }^{15}$ Pai et al also found conversions rates varied for both the TST and the

Table 2 Characteristics of eight longitudinal and serial testing IGRA studies in HCWs

\begin{tabular}{|c|c|c|c|c|c|c|}
\hline Study & Year & Country & $\mathbf{N}$ & $\begin{array}{l}\text { TST } \\
\text { (PPD dose) }\end{array}$ & $\begin{array}{l}\text { IGRA (OFT, TSPOT.TB } \\
\text { or both) }\end{array}$ & $\begin{array}{l}\text { Timing between } \\
\text { repeat testing }\end{array}$ \\
\hline \multicolumn{7}{|c|}{ High TB incidence countries } \\
\hline Pai et $a l^{15}$ & 2006 & India & 216 & $1 \mathrm{TU}$ & QFT-G-IT & 18 months \\
\hline \multicolumn{7}{|c|}{ Intermediate and low TB incidence countries } \\
\hline Pollock et a $\left.\right|^{57}$ & 2009 & USA & $143^{25}$ & $5 \mathrm{TU}$ & QFT-G-IT and T-SPOT.TB & $1-7$ months \\
\hline Zwerling et $\left.a\right|^{59}$ & 2009 & Canada & 117 & 5 TU & OFT-G-IT & 1 year \\
\hline Lee et al, ICHE ${ }^{62}$ & 2009 & Republic of Korea & 196 & 2 TU & QFT-G & 1 year \\
\hline Belknap et $a^{63}$ & 2010 & USA & 1281 & 5 TU & QFT-G-IT and T-SPOT.TB & 6 months \\
\hline Costa et a $\left.\right|^{20}$ & 2010 & Portugal & 670 & 2 TU & QFT-G-IT & $1-2.5$ years \\
\hline Ringshausen et $\left.a\right|^{21}$ & 2010 & Germany & 182 & $2 \mathrm{TU}$ & QFT-G-IT & 18 weeks \\
\hline
\end{tabular}

\footnotetext{
HCW, healthcare worker; IGRA, interferon-gamma release assay; IU, international unit; PPD, purified protein derivative; QFT, QuantiFERON test; QFT-G, QFT Gold test; OFT-G-IT; OFT Gold
} In-Tube test; TB, tuberculosis; TST, tuberculin skin test; TU, tuberculin unit. 


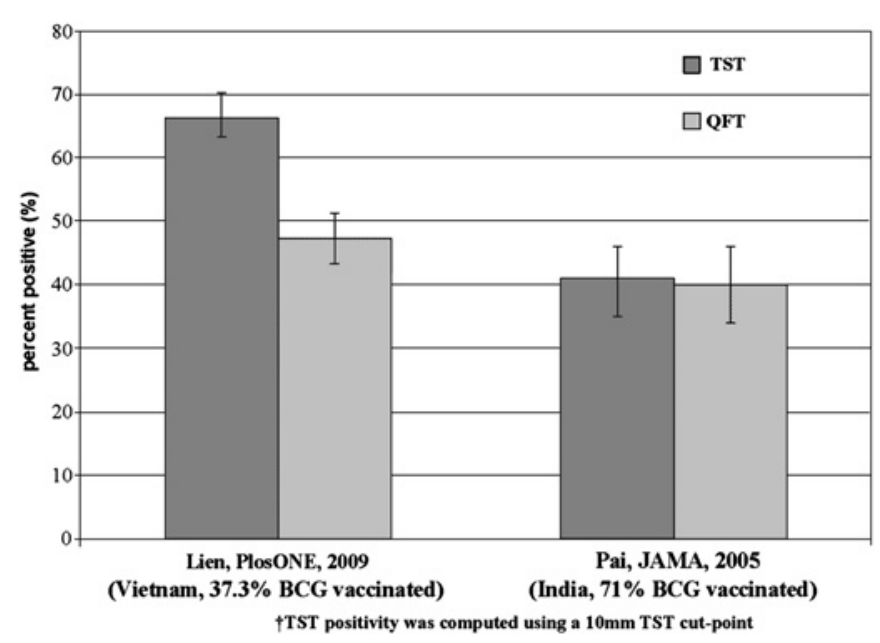

Figure 3 Cross-sectional studies in countries with a high incidence of tuberculosis $(\mathrm{N}=2)$. QFT, QuantiFERON-TB Gold In-Tube; TST, tuberculin skin test.

IGRA when different cut-offs were used. Neither study reported data to suggest that IGRA conversions were better associated with TB exposure than TST conversions.

Reversion rates in the study by Joshi et al ranged from $27 \%$ in the first 6 -month period to $40 \%$ in the second 6-month period. ${ }^{58}$ Pai et al reported 18-month IGRA reversion rates around 7\% among baseline concordant positives, but up to $70 \%$ among those with discordant baseline results (ie, TST-/IGRA + ). ${ }^{15}$

IGRA and TST conversion and reversion rates in HCW populations in low-incidence countries

Four studies have been recently published in this area, while two others were presented at conferences in 2009 and 2010 (table 2). ${ }^{59-64}$ As summarised in table 3 , conversion rates ranged from $1.8 \%(5 / 277)$ in $\operatorname{Japan}^{60}$ (testing every 2 years) to $14.4 \%$ $(21 / 146)$ in Korea ${ }^{62}$ (testing every year). A study by Lee et al ${ }^{62}$ was the only one to report a higher TST conversion rate than IGRA conversion rate (21\% vs $14 \%$ ). Yoshiyama et al in Japan ${ }^{60}$ and Pollock et al in the USA ${ }^{64}$ found IGRA conversions were associated with TB exposure; however, neither performed repeat TST. Belknap et al found IGRA conversion rates were associated with older age and male gender but not occupational exposure to patients with active $\mathrm{TB} .{ }^{63}$ In the only published study to examine the association of IGRA and TST conversions with exposure to $\mathrm{TB},{ }^{62}$ occupational $\mathrm{TB}$ exposure was not associated with TST or IGRA conversions.

Three studies from low- and moderate-incidence settings including Japan, ${ }^{60}$ Canada $^{59}$ and the USA reported IGRA reversion rates of $40-52.9 \%{ }^{63}{ }^{65}$ In the Japanese study, where participants were tested at baseline, at 2 years and at 4 years, all reversions had initial interferon-gamma values close to the cutoff. $^{60}$ This study also showed that OFT conversion was associated with working in a TB ward. A study from Canada found half of the subjects with discordant baseline results (OFT +/TST-) reverted to negative OFT at 1 year. TST was not repeated if the participant was TST + so these studies were not able to estimate TST reversions.

The two most recent serial testing publications in HCWs confirm these early findings. Ringshausen et al in Germany reported a large reversion rate $(6 / 18,33 \%)$ while only $3 / 162$ $(1.9 \%)$ reported IGRA conversions and demonstrated that, when continuous IGRA results were employed, higher baseline results were more stable than those closer to the cut-off. ${ }^{21}$ Costa et al found conversion rates of $3.6-11 \%$ and reversion rates of $5.2-22 \%$ depending on the cut-off used. Using simple negative to positive cut-offs whether for reversions or conversions always gave the largest estimates ( $11 \%$ and $22 \%$, respectively).

Overall, serial testing data from low-incidence countries suggest that IGRA results vary greatly during serial testing, and rates of conversions and reversions vary depending on the test used and the cut-off definition used. When simple negative/ positive changes are used as cut-offs, IGRAs had higher rates of reversions and conversions which were frequently higher than the TST. Owing to the limited number of studies evaluating conversions and a relationship between exposure, there are no data to show that IGRAs are better at identifying the incidence of new TB infection than the TST.
Figure 4 Cross-sectional studies comparing tuberculin skin test (TST) and interferon-gamma release assays (IGRAs) in countries with a low and intermediate incidence of tuberculosis $(\mathrm{N}=24)$ in order of increasing proportion of BCG vaccinated. OFT, QuantiFERON-TB Gold In-Tube.

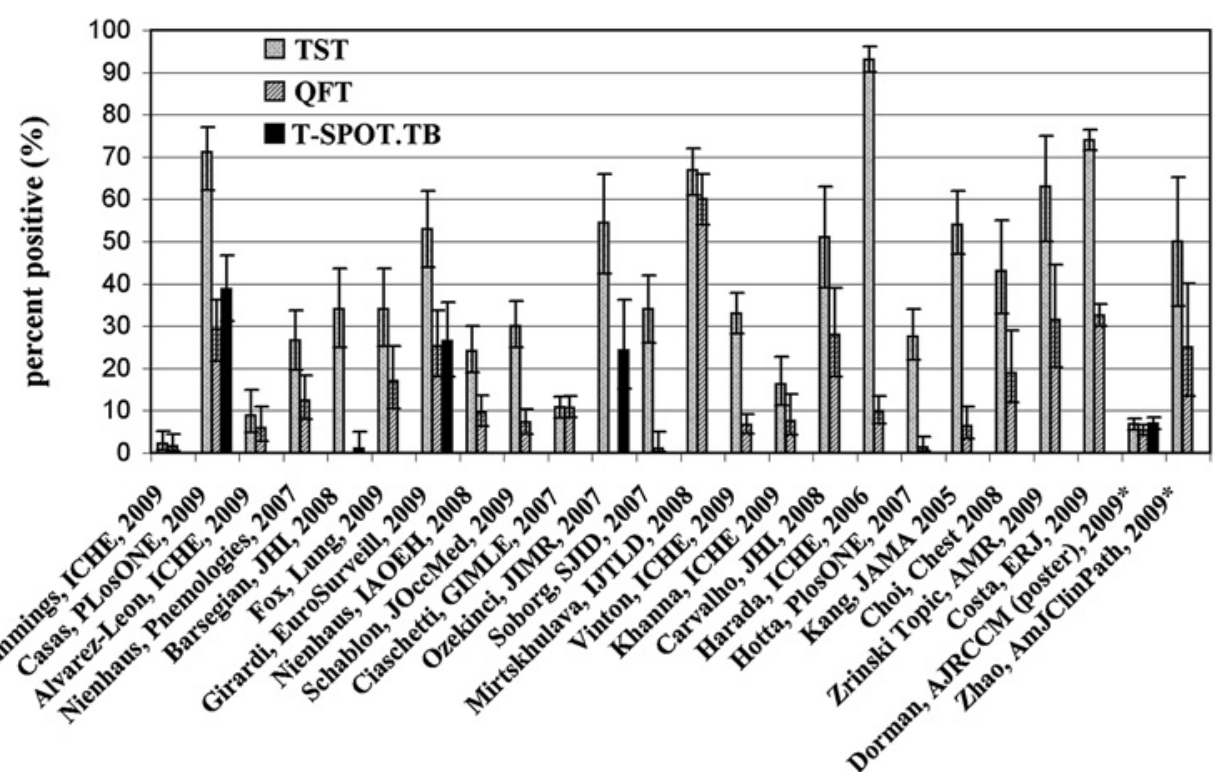

*proportion BCG vaccinated was not reported for these studies

TST positivity was computed using the TST cut-point used by authors of the primary studies. When results for multiple cut-points were presented, data for TST cut-point $10 \mathrm{~mm}$ was displayed 
Figure 5 Occupational exposure to TB as a risk factor for tuberculin skin test (TST) and interferon-gamma release assay (IGRA) positivity: comparison of multivariate ORs. OFT, QuantiFERON-TB Gold In-Tube.

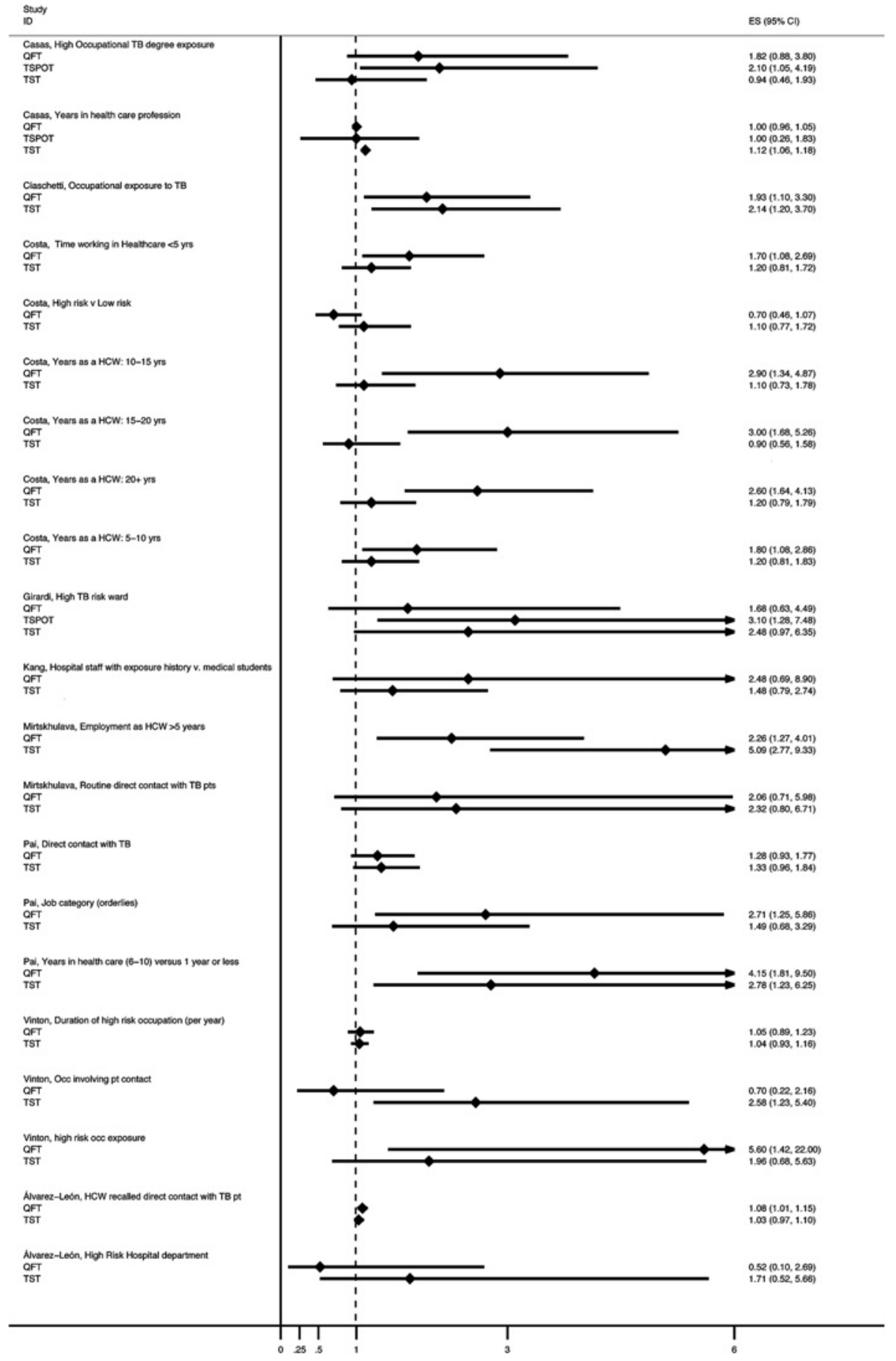

Information on secondary outcomes including cost-effectiveness, feasibility and implementation are available in the online supplement.

\section{DISCUSSION}

While the TST has been successfully used in TBIC programmes, the availability and growing use of IGRAs raises the issue of whether IGRAs could replace the TST for screening of HCWs. Given the repeated nature of routine screening, there are particular issues which may not be relevant in routine practice or contact investigations but become very important when contemplating serial screening. These include test reproducibility, performance of IGRA when repeated frequently, interpretation of discordant TST and IGRA results and the interferon-gamma thresholds (cut-off values) which most accurately distinguish new $\mathrm{TB}$ infection (ie, conversion) from random variation. Despite the paucity of data on the above critical issues, some countries such as the USA have recommended the use of IGRAs for HCW screening. ${ }^{9}$ In contrast, other guidelines (eg, Canada, Australia, UK) ${ }^{17}$ 66-68 have been cautious. In June 2010 the US Centers for Disease Control (CDC) released an updated guideline on IGRAs. ${ }^{69}$ This guideline is more cautious about serial testing with IGRAs and emphasises that using a 'lenient criterion to define IGRA conversion might produce more conversions than are observed with the more stringent criteria applied to TSTs. Furthermore, an association between an IGRA conversion and subsequent disease risk has 
Table 3 Summary of rates of conversions and reversions in serial testing studies $(\mathrm{N}=8)$

\begin{tabular}{|c|c|c|c|c|}
\hline Study & Duration between testing & TST converters, n/N (\%) & IGRA converters, n/N (\%) & IGRA reverters, $\mathrm{n} / \mathrm{N}(\%)$ \\
\hline \multicolumn{5}{|c|}{ High TB incidence countries } \\
\hline Pai, $2006^{15}$ & 18 months & $6 / 147(4.1 \%)$ & $17 / 147(11.6 \%)$ & $7 / 38(18.4 \%)$ \\
\hline \multirow[t]{2}{*}{ Joshi, $2009^{58}$} & 6 months & - & $11 / 57(19 \%)$ & $6 / 22(27 \%)$ \\
\hline & 6 months (6-12 months) & - & $11 / 52^{*}(21 \%)$ & $11 / 27 \dagger(40 \%)$ \\
\hline \multicolumn{5}{|c|}{ Moderate and low TB incidence countries } \\
\hline Pollock, $2009^{64}$ & $1-7$ months & - & $2 / 43 \ddagger(4.6 \%)$ & - \\
\hline Zwerling, $2009^{59}$ & 1 year & $0 / 57(0 \%)$ & $4 / 56(7.14 \%)$ & $4 / 5(80 \%)$ \\
\hline Yoshiyama, $2009^{60}$ & 2 and 4 years & - & $5 / 277(1.8 \%)$ & $13 / 32$ (41\%) \\
\hline Chee, $2009^{61}$ & 1 year & $0 / 18 \ddagger$ & $9 / 182(4.9 \%)$ & - \\
\hline Lee, $2009^{62}$ & 1 year & $16 / 75(21.3 \%)$ & $21 / 146(14.4 \%)$ & - \\
\hline Belknap, $2010^{63}$ & 6 months & $4 / 1202(0.3 \%)$ & $\begin{array}{l}\text { TSPOT } 44 / 1117(3.9 \%) \\
\text { OFT-GIT } 44 / 1169(3.8 \%)\end{array}$ & $\begin{array}{l}\text { TSPOT 36/68 (52.9\%) } \\
\text { OFT-GIT 20/50 (40\%) }\end{array}$ \\
\hline Costa, $2010^{20}$ & $1-2.5$ years & $98 / 199(49.2 \%)$ & $51 / 462(11 \%)$ & $46 / 208(22.1 \%)$ \\
\hline Ringshausen, $2010^{21}$ & 18 weeks & (baseline only) & $3 / 162(1.9 \%)$ & $6 / 18(33.3 \%)$ \\
\hline
\end{tabular}

All conversions/reversions using simple negative/positive definition.

*Denominator includes only participants negative at 6 months.

†Denominator includes only participants positive at 6 months.

$\neq$ Denominator includes only baseline concordant negatives.

IGRA, interferon-gamma release assay; PPD, purified protein derivative; TB, tuberculosis; TST, tuberculin skin test.

not been demonstrated. The criteria for interpreting changes in an IGRA that identify new infections remain uncertain. ${ }^{69}$ Likewise, the most recent 2010 Canadian guidelines also called for caution in the use of IGRAs in serial testing. ${ }^{70}$ In December 2010, the WHO released a STAG report including recommendations which discourage the use of IGRAs, "for the detection of latent $\mathrm{TB}$ infection (LTBI) in adults, children, health-care workers, contacts and those involved in outbreak investigations in low-income and middle-income countries." ${ }^{71}$ This move towards more cautious guidelines in the domain of serial testing is supported by findings in our review, and reinforces the need for an addendum to existing guidelines on IGRAs that specifically address issues related to interpretation of conversions and reversions.

Our systematic review provides several useful insights into the performance of IGRAs in HCWs. The observed prevalence of LTBI in HCWs depends on the test used and the particular TB incidence setting. In low-incidence countries IGRAs estimate a significantly lower prevalence of LTBI than the TST. Some attribute such discordance to the higher specificity of IGRAs compared with the TST and, indeed, agreement and $\kappa$ values are improved in non-BCG-vaccinated individuals compared with BCG-vaccinated, although there is not a strong trend (figure 4).

Studies have suggested that the lower prevalence of LTBI using IGRAs will result in fewer numbers of HCWs who require preventive therapy. However, the higher rate of subsequent conversions found by IGRA in these studies suggests that, while fewer individuals may be identified as LTBI at baseline, more individuals could be diagnosed with conversions by IGRA leading to more HCWs requiring preventive therapy upon repeated screening. This finding has major implications for TBIC policies and relevant cost-effectiveness analyses.

Along with high conversion rates, studies reported high rates of subsequent reversions. This poses concerns of both a scientific and clinical nature. What biological phenomenon is at work here? Are these individuals clearing the infection naturally? Is reinfection an issue in high TB incidence settings? Given a high expected reversion rate, should individuals with positive results be treated, or tested again at a later date? Treating individuals who might have reverted in the absence of treatment could create an environment where more individuals take potentially harmful preventive therapy unnecessarily. This has raised much interest in what is an appropriate definition for an IGRA conversion or reversion.

As summarised in a recent systematic review, ${ }^{18}$ within-subject reproducibility of IGRAs is moderate and a previous TST can potentially boost subsequent IGRA results. IGRAs are dynamic assays and interferon-gamma values tend to fluctuate around the cut-off and cause apparent conversions and reversions. ${ }^{72-74}$ The exact cause of the conversions and reversions remains unclear, and might indicate spontaneous clearance of TB infection, reinfection or dynamic changes within the spectrum of latent $\mathrm{TB}$ infection. ${ }^{75}{ }^{76}$ Conversion rates are highest when a simple negative to positive change is used to define a conversion. This is also true for reversion rates where a simple positive to negative change is used as the definition. Our review suggests that this is true in both high- and low-incidence settings and has implications for deciding on criteria (cut-offs) for conversions and reversions. Alternatives to a simple negative to positive definition for conversion have been proposed by others, including definitions involving an absolute increase over baseline (similar to the TST), a proportionate increase over baseline or a proposed 'grey zone'. ${ }^{73} 74$ These more stringent definitions may lead to smaller rates of conversions and reversions; however, it remains to be seen which conversion definition will be most strongly associated with $\mathrm{TB}$ exposure or subsequent disease development.

Studies found good correlation between occupational risk factors and positivity rates, but very few studies have looked at an association between IGRA test conversion and known occupational exposure or progression to disease. Without these, the interpretation and prognosis of conversions and reversions remains unclear.

\section{Strengths and limitations of the review}

Our systematic review had several strengths. We employed a comprehensive search strategy using multiple sources and databases to retrieve relevant studies, including unpublished studies and conference proceedings. Two review authors independently assessed eligible articles for inclusion. Owing to heterogeneity in study designs and outcomes assessed in each study, it was not appropriate to pool the data, and instead we analysed study results in subgroups by study design and by background TB incidence. 
Despite the comprehensive search, we may have missed relevant studies and publication bias is always a concern. Furthermore, very few studies reported prevalence of HIV among their study populations. Lastly, there is lack of evidence at the highest level of the hierarchy of reference standards; a majority of the included studies were cross-sectional and predominantly from low TB incidence settings. Serial testing data, evidence on predictive value in HCWs and reproducibility data are still quite limited.

\section{Research directions and implications}

Until further evidence is available, TBIC programmes that include IGRA testing must use caution, as emphasised by recent US and Canadian guidelines. ${ }^{69} 70$ In particular, TBIC programmes may observe higher conversion numbers with IGRAs, and health professionals should be cautious about using a simplistic negative-to-positive definition of conversion, and instead consider the amount of change in absolute interferongamma responses as well as relevant clinical information (eg, likelihood of exposure or contact and concurrent TST results, if available) to detect and treat conversions. This is particularly relevant for individuals with borderline results because these results are most likely to change upon retesting.

Current guidelines and evidence available on the use of IGRAs do not adequately address questions raised by serial testing, nor do they provide the guidance or understanding needed to properly interpret IGRA results in serial testing. With rapidly accumulating evidence from serial testing studies, existing IGRA guidelines will need to be updated with specific recommendations on interpretation of serial testing results.

\section{Acknowledgements The authors thank WHO and EG members for their constructive feedback on the review.}

Funding This work was supported in part by USAID funding through TB CAP, the Canadian Institutes of Health Research (grant MOP-81362) and the Special Programme for Research and Training in Tropical Diseases (TDR). These agencies had no role in the design, execution or publication of this study. AZ is supported by a Canadian Institutes of Health Research (CIHR) doctoral research award. MP is a recipient of a CIHR New Investigator Award and DM is a recipient of a Fonds de la recherche en santé du Québec (FRSO) career award.

Competing interests No financial conflicts. At the time this review was conducted MP served as an external consultant for the Foundation for Innovative New Diagnostics (FIND), Geneva, a non-profit agency that works with several industry partners including Cellestis Ltd, Australia in developing and evaluating new diagnostic tools for neglected diseases.

Provenance and peer review Not commissioned; externally peer reviewed.

\section{REFERENCES}

1. Joshi R, Reingold AL, Menzies D, et al. Tuberculosis among health-care workers in low- and middle-income countries: a systematic review. PLoS Med 2006;3:e494.

2. Menzies D, Joshi R, Pai M. Risk of tuberculosis infection and disease associated with work in health care settings. Int J Tuberc Lung Dis 2007;11:593-605.

3. World Health Organization. WHO Policy on TB infection control in health-care facilities, congregate settings and households. Geneva: Stop TB Department, World Health Organization, 2009.

4. Centers for Disease Control and Prevention. Guidelines for preventing the transmission of Mycobacterium tuberculosis in health-care settings. MMWR Recomm Rep 2005;54:1-141.

5. Menzies D. Interpretation of repeated tuberculin tests. Boosting, conversion, and reversion. Am J Respir Crit Care Med 1999;159:15-21.

6. Menzies R. Tuberculin Skin Testing. In: Reichman L, Hershfield E, eds. Tuberculosis: A Comprehensive International Approach. New York: Marcel Dekker, 2000:279-322.

7. Farhat $\mathbf{M}$, Greenaway $\mathrm{C}$, Pai $\mathbf{M}$, et al. False-positive tuberculin skin tests: what is the absolute effect of BCG and non-tuberculous mycobacteria? Int J Tuberc Lung Dis 2006;10:1192-204.

8. Dheda K, Smit RZ, Badri M, et al. T-cell interferon-gamma release assays for the rapid immunodiagnosis of tuberculosis: clinical utility in high-burden vs low-burden settings. Curr Opin Pulm Med 2009;15:188-200.
9. Mazurek GH, Jereb J, Lobue P, et al. Guidelines for using the QuantiFERON-TB Gold test for detecting Mycobacterium tuberculosis infection, United States. MMWR Recomm Rep 2005:54:49-55.

10. Pai M, Riley LW, Colford JM Jr. Interferon-gamma assays in the immunodiagnosis of tuberculosis: a systematic review. Lancet Infect Dis 2004;4:761-76.

11. Pai M, Minion J, Sohn $\mathrm{H}$, et al. Novel and improved technologies for tuberculosis diagnosis: progress and challenges. Clin Chest Med 2009;30:701-16. viii.

12. Menzies D, Pai M, Comstock G. Meta-analysis: new tests for the diagnosis of latent tuberculosis infection: areas of uncertainty and recommendations for research. Ann Intern Med 2007;146:340-54.

13. Pai M, Zwerling A, Menzies D. Systematic review: T-cell-based assays for the diagnosis of latent tuberculosis infection: an update. Ann Intern Med 2008;149:177-84.

14. Diel R, Loddenkemper R, Nienhaus A. Evidence based comparison of commercial interferon-gamma release assays for detecting active tuberculosis: a meta-analysis. Chest 2010;132:952-68.

15. Pai M, Joshi R, Dogra S, et al. Serial testing of health care workers for tuberculosis using interferon-gamma assay. Am J Respir Crit Care Med 2006;174:349-55.

16. Pai M, O'Brien R. Serial testing for tuberculosis: can we make sense of T cell assay conversions and reversions? PLoS Med 2007;4:e208.

17. Canadian Tuberculosis Committee (CTC). Updated recommendations on interferon gamma release assays for latent tuberculosis infection. An Advisory Committee Statement (ACS). CCDR-RMTC 2008;34:1-13.

18. van Zyl-Smit RN, Zwerling A, Dheda K, et al. Within-subject variability of interferon$\mathrm{g}$ assay results for tuberculosis and boosting effect of tuberculin skin testing: a systematic review. PLOS ONE 2009;4:e8517.

19. World Health Organization. WHO Global TB Report. Geneva: World Health Organization, 2009.

20. Torres Costa J, Silva R, Sa R, et al. Serial testing with the interferon-gamma release assay in Portuguese healthcare workers. Int Arch Occup Environ Health 2011;84:461-9.

21. Ringshausen FC, Nienhaus A, Schablon A, et al. Predictors of persistently positive Mycobacterium tuberculosis-specific interferon-gamma responses in the serial testing of health care workers. BMC Infect Dis 2010;10:220.

22. Pai M, Gokhale K, Joshi $\mathrm{R}$, et al. Mycobacterium tuberculosis infection in health care workers in rural India: comparison of a whole-blood, interferon-g assay with tuberculin skin testing. JAMA 2005;293:2746-55.

23. Drobniewski $\mathbf{F}$, Balabanova $Y$, Zakamova $E$, et al. Rates of latent tuberculosis in health care staff in Russia. PLoS Med 2007:4:e55.

24. Lien LT, Hang NT, Kobayashi N, et al. Prevalence and risk factors for tuberculosis infection among hospital workers in Hanoi, Viet Nam. PLOS ONE 2009;4:e6798.

25. Kang YA, Lee HW, Yoon HI, et al. Discrepancy between the tuberculin skin test and the whole-blood interferon gamma assay for the diagnosis of latent tuberculosis infection in an intermediate tuberculosis-burden country. JAMA 2005;293:2756-61.

26. Harada N, Nakajima Y, Higuchi K, et al. Screening for tuberculosis infection using whole-blood interferon-gamma and Mantoux testing among Japanese healthcare workers. Infect Control Hosp Epidemiol 2006;27:442-8.

27. Ozekinci T, Ozbek E, Celik Y. Comparison of tuberculin skin test and a specific T-cell-based test, T-Spot.TB, for the diagnosis of latent tuberculosis infection. J Int Med Res 2007:35:696-703.

28. Veeser PI, Smith PK, Handy B, et al. Tuberculosis screening on a health science campus: use of QuantiFERON-TB Gold test for students and employees. J Am Coll Health 2007:56:175-80.

29. Soborg B, Andersen AB, Larsen HK, et al. Detecting a low prevalence of latent tuberculosis among health care workers in Denmark detected by M. tuberculosis specific IFN-gamma whole-blood test. Scand J Infect Dis 2007;39:554-9.

30. Nienhaus A, Schablon A, Loddenkemper R, et al. [Prevalence of latent tuberculosis infection in healthcare workers in geriatric care]. Pneumologie 2007:61:613-16.

31. Nienhaus A, Loddenkemper R, Hauer B, et al. [Latent tuberculosis infection in healthcare workers-evaluation of an Interferon-gamma release assay]. Pneumologie 2007:61:219-23.

32. Mirtskhulava V, Kempker R, Shields KL, et al. Prevalence and risk factors for latent tuberculosis infection among health care workers in Georgia. Int J Tuberc Lung Dis 2008; 12:513-19.

33. Hotta K, Ogura T, Nishii K, et al. Whole blood interferon-gamma assay for baseline tuberculosis screening among Japanese healthcare students. PLoS ONE 2007;2:e803.

34. Nienhaus A, Schablon A, Bacle CL, et al. Evaluation of the interferon-gamma release assay in healthcare workers. Int Arch Occup Environ Health 2008;81:295-300.

35. Ciaschetti A, Franchi A, Richeldi $L$, et al. [Screening of latent tuberculosis infection in health care workers by QuantiFERON-TB and tuberculin skin test]. G Ital Med Lav Ergon 2007;29(Suppl 3):406-7.

36. Eum SY, Lee YJ, Kwak HK, et al. Evaluation of the diagnostic utility of a whole-blood interferon-gamma assay for determining the risk of exposure to Mycobacterium tuberculosis in Bacille Calmette-Guerin (BCG)-vaccinated individuals. Diagn Microbiol Infect Dis 2008:61:181-6.

37. Choi JC, Shin JW, Kim JY, et al. The effect of previous tuberculin skin test on the follow-up examination of whole-blood interferon-gamma assay in the screening for latent tuberculosis infection. Chest 2008;133:1415-20.

38. Carvalho AC, Crotti N, Crippa M, et al. QuantiFERON((R))-TB Gold test for healthcare workers. J Hosp Infect 2008;69:91-2.

39. Barsegian V, Mathias KD, Wrighton-Smith P, et al. Prevalence of latent tuberculosis infection in German radiologists. J Hosp Infect 2008;69:69-76. 
40. Stebler A, Iseli P, Muhlemann K, et al. Whole-blood interferon-gamma release assay for baseline tuberculosis screening of healthcare workers at a Swiss university hospital. Infect Control Hosp Epidemiol 2008;29:681-3.

41. Thijsen SF, van Rossum SV, Arend S, et al. The value of interferon gamma release assays for diagnosis infection with mycobacterium tuberculosis during an annual screening of health care workers. J Occup Environ Med 2008:50:1207-8.

42. Demkow U, Broniarek-Samson B, Filewska M, et al. Prevalence of latent tuberculosis infection in health care workers in Poland assessed by interferon-gamma whole blood and tuberculin skin tests. J Physiol Pharmacol 2008;59(Suppl 6):209-17.

43. Schablon A, Beckmann G, Harling M, et al. Prevalence of latent tuberculosis infection among health care workers in a hospital for pulmonary diseases. J Occup Med Toxicol 2009;4:1

44. Dorman S, Belknap R, Weinfurter $P$, et al. Evaluation of new interferon-gamma release assays (IGRAs) in the diagnosis of latent tuberculosis infection in U.S. health care workers: baseline testing results. Am J Respir Crit Care Med 2009:A1010.

45. Mehta SR, MacGruder C, Looney D, et al. Differences in tuberculin reactivity as determined in a veterans administration employee health screening program. Clin Vaccine Immunol 2009;16:541-3.

46. Vinton $\mathbf{P}$, Mihrshahi S, Johnson P, et al. Comparison of QuantiFERON-TB Gold in-tube test and tuberculin skin test for identification of latent Mycobacterium tuberculosis infection in healthcare staff and association between positive test results and known risk factors for infection. Infect Control Hosp Epidemiol 2009;30:215-21.

47. Topic RZ, Dodig S, Zoricic-Letoja I. Interferon-gamma and immunoglobulins in latent tuberculosis infection. Arch Med Res 2009:40:103-8.

48. Khanna $\mathbf{P}$, Nikolayevskyy V, Warburton F, et al. Rate of latent tuberculosis infection detected by occupational health screening of nurses new to a London teaching hospital. Infect Control Hosp Epidemiol 2009;30:581-4.

49. Alvarez-Leon EE, Espinosa-Vega E, Santana-Rodriguez E, et al. Screening for tuberculosis infection in spanish healthcare workers: comparison of the QuantiFERON-TB Gold in-tube test with the tuberculin skin test. Infect Control Hosp Epidemiol 2009;30:876-83.

50. Casas I, Latorre I, Esteve $\mathrm{M}$, et al. Evaluation of interferon-gamma release assays in the diagnosis of recent tuberculosis infection in health care workers. PLOS ONE 2009; 4:e6686

51. Fox BD, Kramer MR, Mor Z, et al. The QuantiFERON((R))-TB-Gold assay for tuberculosis screening in healthcare workers: a cost-comparison analysis. Lung 2009;187:413-19.

52. Costa JT, Sa R, Cardoso MJ, et al. Tuberculosis screening in Portuguese healthcare workers using the tuberculin skin test and the interferon-gamma release assay. Eur Respir J 2009;34:1423-8.

53. Costa JT, Silva $\mathrm{R}$, Sa $\mathrm{R}$, et al. Comparison of interferon-gamma release assay and tuberculin test for screening in healthcare workers. Rev Port Pneumol 2010;16:211-21.

54. Zhao X, Mazlagic D, Flynn EA, et al. Is the QuantiFERON-TB blood assay a good replacement for the tuberculin skin test in tuberculosis screening? A pilot study at Berkshire Medical Center. Am J Clin Pathol 2009;132:678-86.

55. Girardi E, Angeletti C, Puro V, et al. Estimating diagnostic accuracy of tests for latent tuberculosis infection without a gold standard among healthcare workers. Euro Surveill 2009;14:19373

56. Cummings KJ, Smith TS, Shogren ES, et al. Prospective comparison of tuberculin skin test and QuantiFERON-TB Gold In-Tube assay for the detection of latent tuberculosis infection among healthcare workers in a low-incidence setting. Infect Control Hosp Epidemiol 2009;30:1123-6.

57. Pollock NR, Campos-Neto A, Kashino S, et al. Discordant QuantiFERON-TB Gold test results among US healthcare workers with increased risk of latent tuberculosis infection: a problem or solution? Infect Control Hosp Epidemiol 2008:29:878-86.
58. Joshi R, Gajalakshmi D, Reddy MVR, et al. Unstable IGRA Conversions and Reversions over Three Time Points among Medical Students in a High Burden Setting Dubrovnik, Croatia: 2nd Global Symposium on IGRAs, 2009.

59. Zwerling A, Cloutier-Ladurantaye J, Pietrangelo F, et al. Conversions and reversions in health care workers in Montreal, Canada using QuantiFERON-TB-Gold In-Tube. Am J Respir Crit Care Med 2009:A1012.

60. Yoshiyama T, Harada N, Higuchi K, et al. Estimation of incidence of tuberculosis infection in health-care workers using repeated interferon-gamma assays. Epidemio Infect 2009:137:1691-8.

61. Chee CB, Lim LK, Barkham TM, et al. Use of a T cell interferon-gamma release assay to evaluate tuberculosis risk in newly qualified physicians in Singapore healthcare institutions. Infect Control Hosp Epidemiol 2009;30:870-5.

62. Lee K, Han MK, Choi HR, et al. Annual incidence of latent tuberculosis infection among newly employed nurses at a tertiary care university hospital. Infect Control Hosp Epidemiol 2009;30:1218-22.

63. Belknap R, Wall K, Teeter L, et al. Interferon-gamma release assays (IGRAs) in seria testing for latent tuberculosis infection in U.S. health care workers. Am J Respir Crit Care Med 2010:A2263.

64. Pollock NR, Kashino SS, Napolitano DR, et al. Evaluation of the effect of treatment of latent tuberculosis infection on QuantiFERON-TB Gold assay results. Infect Control Hosp Epidemiol 2009;30:392-5.

65. Belknap R, Feske M, Choung G, et al. Diagnosis of Latent Tuberculosis Infection in Health Care Workers: Impact of a recent Tuberculin Skin Test on the Interferongamma Release Assays (IGRAs). American Thoracic Society 2009; Sunday May 17 2009. San Diego, USA. Am J Respir Crit Care Med 2009:A1011.

66. National Institute for Health and Clinical Excellence (NICE). Tuberculosis: clinical diagnosis and management of tuberculosis, and measures for its prevention and control. Clinical Guideline 33. London: NICE, 2006. http://www.nice.org.uk/page. aspx?o=271310, http://www.nice.org.uk/page.aspx?o=CG033NICEguideline

67. Australian National Tuberculosis Advisory Committee. Position statement on interferon-gamma release immunoassays in the detection of latent tuberculosis infection. Commun Dis Intell 2007;31:404-5.

68. HPA Tuberculosis Programme Board. Health Protection Agency Position Statement on the use of Interferon Gamma Release Assay (IGRA) tests for tuberculosis (TB): draft for consultation, October 2007. London: Health Protection Agency, 2007.

69. Mazurek M, Jereb J, Vernon A, et al. Updated guidelines for using interferon gamma release assays to detect Mycobacterium tuberculosis infection - United States. MMWR Recomm Rep 2010;59:1-25.

70. Canadian Tuberculosis Committee (CTC). Recommendations on interferon gamma release assays for the diagnosis of latent tuberculosis infection -2010 update. Can Commun Dis Rep 2010:36.

71. World Health Organization. Strategic and Technical Advisory Group for Tuberculosis (STAG-TB). Report of the Tenth Meeting. Geneva: WHO, 2010

72. Detjen AK, Loebenberg L, Grewal HM, et al. Short-term reproducibility of a commercial interferon gamma release assay. Clin Vaccine Immunol 2009;16:1170-5

73. van Zyl-Smit RN, Pai M, Peprah K, et al. Within-subject variability and boosting of T-cell interferon-gamma responses after tuberculin skin testing. Am J Respir Crit Care Med 2009;180:49-58.

74. Veerapathran A, Joshi R, Goswami K, et al. T-cell assays for tuberculosis infection: deriving cut-offs for conversions using reproducibility data. PLOS ONE 2008:3:e1850.

75. Barry CE 3rd, Boshoff HI, Dartois V, et al. The spectrum of latent tuberculosis: rethinking the biology and intervention strategies. Nat Rev Microbiol 2009;7:845-55.

76. Pai M. Spectrum of latent tuberculosis: existing tests cannot resolve the underlying phenotypes. Nat Rev Microbiol 2010:8:242.

\section{Thorax Online Archive}

Visit our Online Archive - available back to 1946. Subscribers may access the entire archive freely. Non-subscribers have free access to all articles prior to 2006. A simple one-time registration is required that grants access to all the free archive content, across all of our specialist titles. To view or to register visit thorax.bmj.com. 